\section{Novel Serotype of Epizootic Hemorrhagic Disease Virus, China}

\author{
Heng Yang, ${ }^{1}$ Zhuoran $\mathrm{Li},{ }^{1}$ Jinping Wang, \\ Zhanhong Li, Zhenxing Yang, Defang Liao, \\ Jianbo Zhu, Huachun Li
}

Author affiliation: Yunnan Animal Science and Veterinary Institute, Kunming, China

DOI: https://doi.org/10.3201/eid2612.191301

In 2018, a strain of epizootic hemorrhagic disease virus (EHDV), named YNDH/V079/2018, was isolated from a sentinel calf in Mangshi County, Yunnan Province, China. Nucleotide sequencing and neutralization tests indicated that the virus belongs to a novel serotype of EHDV that had not been reported previously.

E pizootic hemorrhagic disease virus (EHDV; family Reoviridae, genus Orbivirus) is transmitted between ruminants by Culicoides spp. biting midges. It is widespread in tropical and subtropical regions and primarily infects white-tailed deer and cattle (1); EHDV infection often causes death in white-tailed deer. Seven serotypes of EHDV (EHDV-1, -2, -4, -5, -6, -7, and -8) have been officially assigned; EHDV-3 (NIG1967/01 strain) has been combined into EHDV-1 $(1,2)$. Recently, 2 novel EHDV strains isolated from South Africa (3) and Japan (4) were suggested as new serotype candidates. Although only Ibaraki virus (a strain of EHDV-2 from Japan) was previously known to cause a bluetongue-like illness in cattle (1), EHDV-1, EHDV-2, EHDV-6, and EHDV-7 have recently been associated with illness and death in cattle in Asia, the Mediterranean Basin, South Africa, North America, and Réunion Island (1,5-7), suggesting that the distribution and the pathogenicity associated with EHDV infection are increasing. EHDVs belonging to serotypes 1, 5, 6, and 7, as well as a nontyped serotype, have been isolated from sentinel cattle in southern China (H. Li et al., unpub. data).

In 2018, we placed 10 Yunnan yellow cattle 6-12 months of age and free of EHDV antibodies under field conditions at Sanjiaoyan village, Mangshi County, Dehong Prefecture, Yunnan Province, China (Appendix Figure 1, https://wwwnc.cdc.gov/EID/ article/26/12/19-1301-App1.pdf) as sentinel animals. We took blood samples at weekly intervals during May-October: whole blood for serology, EDTA samples for viral nucleic acid detection, and heparin blood samples for virus isolation.

${ }^{1}$ These authors contributed equally to this article.
During June-September, we confirmed EHDV infections in 3 of the sentinel cattle by EHDV competitive ELISA (cELISA; ID-Vet, https:/ / www.id-vet.com) and real-time quantitative reverse transcription PCR (qRTPCR) (3). We isolated viruses from blood samples by inoculating C6/36 cells and blindly passaging for 5 times on BHK-21 cells (1). We isolated EHDV-1 and EHDV-5 strains from 2 of the cattle and an additional strain of EHDV, YNDH/V079/2018, from the third animal. Serotype identification of YNDH/V079/2018 displayed uniform negative results through serotype-specific RT-PCR (8) and virus neutralization tests using serum samples against EHDV-1, EHDV-2, EHDV-5, EHDV6, EHDV-7, EHDV-8, and nontyped serotype reference strains. Furthermore, serum from the YNDH/ V079/2018-infected calf showed no neutralization to tested serotypes of EHDV reference strains.

Double-stranded RNA extracted from YNDH/ V079/2018 generated a genome segment migration pattern typical of bluetongue virus or EHDV (Appendix Figure 2) by agarose gel electrophoresis. Transmission electron microscopy revealed virus particles $\approx 80$ $\mathrm{nm}$ in diameter, with a ring-shaped capsomere characteristic of Orbivirus (Appendix Figure 3). Full-length cDNA copies of segments 2 and 3 (Seg-2 and Seg-3) of YNDH/V079/2018 were synthesized and sequenced as described by Maan et al. (9). BLAST analyses (https://www.ncbi.nlm.nih.gov/BLAST) of Seg-2 and Seg-3 sequences (GenBank accession nos. MN418446 and MN418447) revealed the highest matching identities with equivalent genome segments of other EHDVs.

Subcore shell viral protein (VP) 3 of EHDV, encoded by Seg-3, is highly conserved, showing $>95.5 \%$ amino acid sequence identity within EHDVs (10). Seg-3/VP3 of YNDH/V079/2018 showed overall nucleotide/amino acid identity levels of $78.5 \% / 94.6 \%$ to 80.0\% /96.5\% with other EHDVs, confirming its identity as an EHDV isolate. However, Seg-3 of YNDH/ V079/2018 did not cluster with previously identified Eastern or Western EHDV topotypes (10); maximum sequence identities were $80.0 \% \mathrm{nt}$ and $78.9 \%$ aa, which placed YNDH/V079/2018 as a distinct topotype in the phylogenetic tree (Figure, panel A).

The outer capsid protein VP2 of EHDV, encoded by Seg-2, is highly variable and is definitive for serotype determination (2). Seg-2/VP2 of $\mathrm{YNDH} /$ V079/2018 sharing sequence identities of $44.3 \%$ $50.9 \%$ nt and $31.0 \%-40.6 \%$ aa to previously recognized EHDV serotypes, which supports it as a distinct EHDV Seg-2 group (2), herein named group E (Figure, panel B). This finding coincides with the results of neutralization tests and indicates that YNDH/ V079/2018 represents a novel serotype of EHDV. 

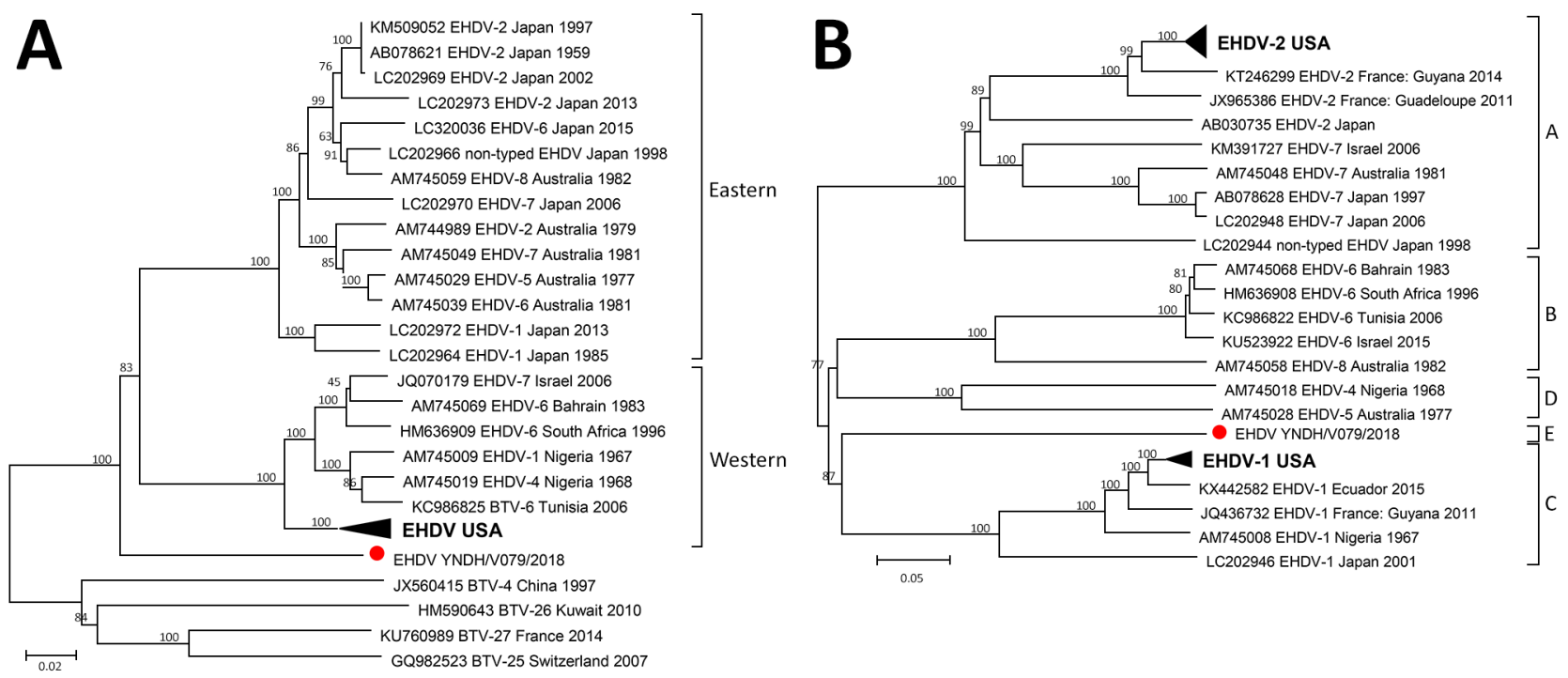

Figure. Phylogenetic analyses of EHDV based on segment 3 (A) and segment 2 (B) of YNDH/V079/2018 from Mangshi County, Yunnan Province, China (red dot), compared with other global EHDV isolates. The following convention was used to identify sequences: GenBank accession no., EHDV-serotype, country, isolation year. Eastern and Western topotypes of segment 3 and A-D groups of segment 2 were assigned as described by Anthony et al. (2,10); a distinct segment 2 group of the strain YNDH/V079/2018 isolated in China (2) is marked as group E. The nontyped strain from Japan isolated in 1998 is included in accession nos. LC202966 and LC202944 (4). We did not include the nontyped strain from South Africa, due to the lack of sequence information in GenBank. BTV strains were used as the outgroups. Number at each branch indicates a bootstrap value. Scale bars indicate nucleotide substitutions per site. BTV, bluetongue virus; EHDV, epizootic hemorrhagic disease virus.

We developed conventional RT-PCR and qRTPCR targeting Seg-2 of YNDH/V079/2018 (Appendix Table 1) and used them in combination with cELISA and serum neutralization tests to trace progress of the infection in the sentinel animal (Appendix Table 2). We defined the earliest week in which the virus was detectable in the blood using qRT-PCR as infection week 1. We isolated YNDH/V079/2018 in week 2 from the sentinel calf, which experienced fever to $40.2^{\circ} \mathrm{C}$, anorexia, and respiratory distress. Viral nucleic acid in the blood peaked in weeks 1-2; levels decreased gradually until none was detected at week 14. cELISA and neutralization antibodies first appeared at week 2, peaked in weeks 7-8, and remained elevated until week 17 , when blood sampling ceased.

To determine the prevalence of YNDH/ V079/2018, we tested 87 EDTA cattle blood samples from 3 farms in Mangshi County during April-October 2018 but detected no nucleic acid consistent with YNDH/V079/2018. Additional genome sequencing, type-specific diagnostic tests, and epidemiologic and pathogenic investigations of this novel EHDV are planned. Our study will help clarify the diversity of EHDV serotypes and the distribution and pathogenicity of this novel EHDV and its potential risk to ruminants.

\section{Acknowledgments}

We thank colleagues from the Center for Animal Disease Control and Prevention of Dehong Prefecture who collected blood samples from sentinel animals.

This study, "Arbovirus Disease Research in Cattle and Sheep," was supported by Special Fund for Agro-scientific Research in the Public Interest (project no. 201303035) and National Key R\&D Program of China (project no. 2017YFC1200500).

\section{About the Author}

Dr. Yang is a research fellow in Yunnan Animal Science and Veterinary Institute, Kunming, China. His current research interests include characterization of bluetongue virus and other related arboviruses of ruminants.

\section{References}

1. World Organisation for Animal Health. Epizootic hemorrhagic disease (infection with epizootic hamorrhagic disease virus). In: Manual of diagnostic tests and vaccines for terrestrial animals 2019. Paris; The Organisation: 2018. p. 422-32.

2. Anthony SJ, Maan S, Maan N, Kgosana L, Bachanek-Bankowska K, Batten C, et al. Genetic and phylogenetic analysis of the outer-coat proteins VP2 and VP5 of epizootic haemorrhagic disease virus (EHDV): comparison of genetic and serological data to characterise the EHDV serogroup. Virus Res. 2009;145:200-10. https://doi.org/10.1016/j.virusres.2009.07.012 
3. Maan NS, Maan S, Potgieter AC, Wright IM, Belaganahalli M, Mertens PPC. Development of real-time RT-PCR assays for detection and typing of epizootic haemorrhagic disease virus. Transbound Emerg Dis. 2017;64:1120-32. https://doi.org/10.1111/tbed.12477

4. Shirafuji H, Kato T, Yamakawa M, Tanaka T, Minemori Y, Yanase T. Characterization of genome segments 2, 3 and 6 of epizootic hemorrhagic disease virus strains isolated in Japan in 1985-2013: identification of their serotypes and geographical genetic types. Infect Genet Evol. 2017;53:38-46. https://doi.org/10.1016/j.meegid.2017.05.010

5. Golender N, Bumbarov VY. Detection of epizootic hemorrhagic disease virus serotype 1, Israel. Emerg Infect Dis. 2019;25:825-7. https:/ / doi.org/10.3201/ eid2504.180149

6. Temizel EM, Yesilbag K, Batten C, Senturk S, Maan NS, Mertens PPC, et al. Epizootic hemorrhagic disease in cattle, western Turkey. Emerg Infect Dis. 2009;15:317-9. https:// doi.org/10.3201/eid1502.080572

7. Kamomae Y, Kamomae M, Ohta Y, Nabe M, Kagawa Y, Ogura Y, et al. Epizootic hemorrhagic disease virus serotype 6 infection in cattle, Japan, 2015. Emerg Infect Dis. 2018;24:902-5. https://doi.org/10.3201/eid2405.171859

8. Maan NS, Maan S, Nomikou K, Johnson DJ, El Harrak M, Madani H, et al. RT-PCR assays for seven serotypes of epizootic haemorrhagic disease virus \& their use to type strains from the Mediterranean region and North America. PLoS One. 2010;5:e12782. https://doi.org/10.1371/ journal.pone.0012782

9. Maan S, Rao S, Maan NS, Anthony SJ, Attoui H, Samuel AR, et al. Rapid cDNA synthesis and sequencing techniques for the genetic study of bluetongue and other dsRNA viruses. J Virol Methods. 2007;143:132-9. https://doi.org/10.1016/ j.jviromet.2007.02.016

10. Anthony SJ, Maan N, Maan S, Sutton G, Attoui H, Mertens PP. Genetic and phylogenetic analysis of the core proteins VP1, VP3, VP4, VP6, and VP7 of epizootic haemorrhagic disease virus (EHDV). Virus Res. 2009; 145:187-99. https://doi.org/10.1016/j.virusres.2009.07.011

Address for correspondence: Huachun Li or Heng Yang, Yunnan Animal Science and Veterinary Institute, Jindian St, Kunming 650224, China; email: Li_huachun@hotmail.com or yangheng2008.cool@163.com

\section{Pathogenic New World Relapsing Fever Borrelia in a Myotis Bat, Eastern China, 2015}

Hui-Ju Han, Jian-Wei Liu, Hong-Ling Wen, Ze-Min Li, Si-Cong Lei, Xiang-Rong Qin, Chuan-Min Zhou, Hao Yu, Xiao Xiao, Xue-Jie Yu

Author affiliations: Wuhan University, Wuhan, China (H.-J. Han, J.-W. Liu, Z.-M. Li, S.-C. Lei, X.-R. Qin, C.-M. Zhou, H. Yu, X.-J. Yu); Shandong University, Jinan, China (H.-L. Wen); Hubei University of Chinese Medicine, Wuhan (X. Xiao)

DOI: https://doi.org/10.3201/eid2612.191450

We identified Candidatus Borrelia fainii, a human pathogenic bacterium causing New World relapsing fever in a Myotis bat in eastern China. This finding expands knowledge about the geographic distribution of Borrelia spp. and the potential for infection with New World relapsing fever in China.

Rorrelia is a genus comprising 3 groups of spiro$\mathrm{B}_{\text {chetes: the Lyme disease group, the relapsing fever }}$ group, and a nonconformist third group. Typically, Lyme disease borreliae are transmitted by hard ticks and have a worldwide distribution, but most relapsing fever Borrelia are transmitted by soft ticks, except for louse-borne B. recurrentis. Relapsing fever borreliae are further classified into 2 subgroups, New World relapsing fever (NWRF) Borrelia and Old World relapsing fever, on the basis of epidemic regions and the genetic lineage of the causative agent. $B$. lonestari and B. miyamotoi are transmitted by hard ticks, but are more closely related to relapsing fever borreliae than to Lyme disease borreliae and are distributed both in the New World (North and South America and Oceania) and the Old World (Europe, Asia, and Africa). The nonconformist third group includes an orphan Borrelia species named B. turicata (1). We identified Candidatus Borrelia fainii in a Myotis bat from eastern China.

During March-October 2015, with the help of local farmers, we caught a total of 145 bats from various niches in Mengyin County, Shandong Province, China, using mist nets or butterfly nets and took tissue samples (liver, spleen, lung, or kidney). The captured bats included 4 Rhinolophus ferrumequinum and 14 Rh. pusillus from a karst cave, 26 Eptesicus serotinus from 2 farm houses, 34 Myotis fimbriatus and $10 \mathrm{M}$. ricketti from a city sewer, and $57 \mathrm{M}$. pequinius from a cave. We extracted DNA from the bat tissue, then screened for Borrelia by amplifying the $r r s, f l a B$, and $g l p Q$ genes using methods 